Article

\title{
Spray-Dried Amorphous Solid Dispersions of Atorvastatin Calcium for Improved Supersaturation and Oral Bioavailability
}

\author{
Jaewook Kwon ${ }^{\dagger}$, Bhupendra Raj Giri ${ }^{\dagger}$, Eon Soo Song, Jinju Bae, Junseong Lee and \\ Dong Wuk Kim * iD \\ College of Pharmacy \& Research Institute of Pharmaceutical Sciences, Kyungpook National University, \\ Daegu 41566, Korea \\ * Correspondence: dkim17@knu.ac.kr; Tel.: +82-53-950-8579 \\ + These authors contributed equally to this work.
}

Received: 8 August 2019; Accepted: 4 September 2019; Published: 6 September 2019

check for updates

\begin{abstract}
Over the past few decades, the amorphous solid dispersions (ASDs) technique has emerged as a promising strategy to enhance the in vitro/in vivo characteristic of hydrophobic drugs. The low aqueous solubility and poor bioavailability of atorvastatin calcium (ATO), a lipid-lowering drug, present challenges for effective drug delivery. The objective of this work was to improve the aqueous solubility, in vitro dissolution, and oral absorption of ATO with amorphous solid dispersion technique prepared by spray-drying method. The optimized ternary formulation comprising of ATO; hydroxypropyl methylcellulose (HPMC), as a hydrophilic polymer; and sodium lauryl sulfate (SLS), as a surfactant, at a weight ratio of $1 / 1 / 0.1$, showed significant improvement in aqueous solubility by 18 -fold compared to that of the free drug, and a cumulative release of $94.09 \%$ compared to a release of $59.32 \%$ of the free drug. Further, physicochemical studies via scanning electron microscopy, differential scanning calorimetry, and powder X-ray diffraction revealed a change from the crystalline state of the free drug to its amorphous state in the ASD. Pharmacokinetic analysis in rats demonstrated 1.68- and 2.39-fold increments in AUC and $C_{\max }$, respectively, in the ASD over the free drug. Altogether, hydrophilic carrier-based ASDs prepared by the spray-drying technique represent a promising strategy to improve the biopharmaceutical performance of poorly soluble drugs.
\end{abstract}

Keywords: atorvastatin calcium; hydroxypropyl methylcellulose (HPMC); amorphous solid dispersion; spray-drying; solubility enhancement; bioavailability

\section{Introduction}

Approximately $40 \%$ of new chemical entities identified in drug discovery programs have poor aqueous solubility [1,2]. Although these chemical entities possess potentially desirable pharmacodynamic properties, they fail to reach the market due to their low bioavailability. Over the past few decades, several methods have been employed to improve drug solubility, including salt formation [3], use of prodrug strategies [4], particle size reduction (micronization and nanonization) [5], complexation [6], use of lipid based formulations [7], and use of solid dispersions [8-10].

Solid dispersion (SD), a concept introduced by Sekiguchi and Obi in the 1960s [11,12], is the dispersion of a hydrophobic drug in one or more hydrophilic carrier (matrix), where the drug can be present in a solubilized, fine crystalline, or amorphous state [13]. Today, SD has become one of the most widely accepted and promising approaches for improving drug solubility leading to enhanced bioavailability [14]. Increase in surface area via particle size reduction, molecular dispersion of crystalline compounds into hydrophilic carriers resulting in improved wettability and enhanced 
porosity, and manipulation of the solid state of the drug substance, i.e., the transformation of a crystalline drug to its amorphous state, all of which result from use of SD systems, leading to improved in vitro and in vivo drug characteristics [15]. Common methods used to prepare ASDs include quench cooling from the melt, rapid solvent evaporation, melt extrusion, spray-drying, and electrospinning/spraying. Spray-drying is a commonly used solvent evaporation technique for the production of SDs and is extensively used in pharmaceutical industry. It mainly involves dissolving or suspending or drug and carrier(s), atomization of the solution/suspension, mixing and drying of liquid with a stream of heated air, and separation of the dried particles from the hot gas [16].

The carriers used for drug encapsulation have a major influence on the release profile of the dispersed drug. Such carriers include hydroxypropyl cellulose, hydroxypropyl methylcellulose (HPMC), ethyl cellulose, cellulose acetate phthalate, ethyl acetate, chitosan, and methacrylic acid copolymers [17]. HPMC is an attractive nonionic, water-soluble, cellulose ether derivative. It is a semisynthetic and viscoelastic cellulose molecule associated with ease of manufacturing of oral solid dosage forms (OSDFs), and is accepted globally by regulatory bodies [18].

The mechanism of drug release from solid dispersions containing HPMC matrix involves a complex phenomenon [19], typically involving wetting, liquid penetration into the dry core matrix, polymer gelatinization (polymer dissolution in a semidilute state), polymer dissolution, and diffusion/erosion of the solubilized drug through the gel layer. Concomitantly, outer layers of the SD system become fully hydrated and dissolve, referred to as erosion, eventually releasing the encapsulated drug. As the outer layer undergoes complete hydration and dissolution, the inner layers begin to continuously replace it [20] (Figure 1). Generally it is presumed that the hydrophilic drugs are released mainly through diffusion from the HPMC gel layer whereas, the hydrophobic drugs are largely released via erosion of the gel layer [21]. These processes are mainly affected by interactions of the drug with the hydrophilic polymer used for incorporation and formation of a drug-loaded carrier matrix.

A third-generation SD system is developed with the addition of a third agent or more excipients along with a polymer carrier (so called ternary SD), in order to prevent drug recrystallization during storage. These third agents commonly include surfactants such as, polyethylene-polypropylene glycol, bile salt, poloxamers, polysorbates, sodium lauryl sulfate (SLS), alkyl benzene sulfonates, benzalkonium chloride, etc., which can improve the solubility and dissolution process and stabilize the amorphous form of drug in SD. Further, the surfactants can behave as a precipitation inhibitor to further increase the degree of supersaturation in vivo [22,23]. Therefore, careful polymer and surfactant selection is crucial in gaining the maximum advantage of ASDs and avoiding failure through drug recrystallization.

Atorvastatin calcium is a synthetic lipid-lowering drug, generally prescribed in the treatment atherosclerosis and coronary disease with or without other lipid lowering agents [24,25]. ATO is the most preferred drug candidate among other statins that are indicated for treatment of moderate to severe familial or non-familial hypercholesterolemia [26,27]. LIPITOR ${ }^{\circledR}$ tablets (brand of atorvastatin calcium) are commercially available in different strengths, including 10, 20, 40 or $80 \mathrm{mg}$. ATO as a drug candidate was selected because of its low oral bioavailability ( $12 \%$ from a $40 \mathrm{mg}$ oral dosage form), poor aqueous solubility, crystalline nature, and high hepatic first-pass metabolism $[28,29]$. Thus, to gain maximum pharmacotherapeutic actions, the aqueous solubility of ATO needs to be improved. We hypothesized that the in vitro and in vivo characteristics of atorvastatin calcium (ATO) would be improved by incorporating the drug into a cellulose matrix. Several studies have been carried out with ATO, such as preparation of ATO SDs [30-32], size reduction (i.e., ATO nanopreparations) [33]. However, the influence of the cellulose matrix system on the preparation and characterization ATO-loaded ternary solid dispersion system is an area of interest that needs investigating. Therefore, the overall objective of the present study is to investigate the influence of a cellulose polymer in an atorvastatin calcium (ATO)-loaded ternary amorphous solid dispersion system with the goal of improving aqueous solubility and dissolution profile, and thus enhancing the oral bioavailability of the drug. The ATO-ASDs comprising the drug, HPMC, and sodium lauryl sulfate (SLS) were prepared via 
a spray-drying technique. The solid dispersions were characterized by scanning electron microscopy (SEM), differential scanning calorimetry (DSC), and X-ray powder diffraction (XRD). The dissolution profiles of the formulations were assessed in vitro, while the oral bioavailability of ASD was quantified in rats.

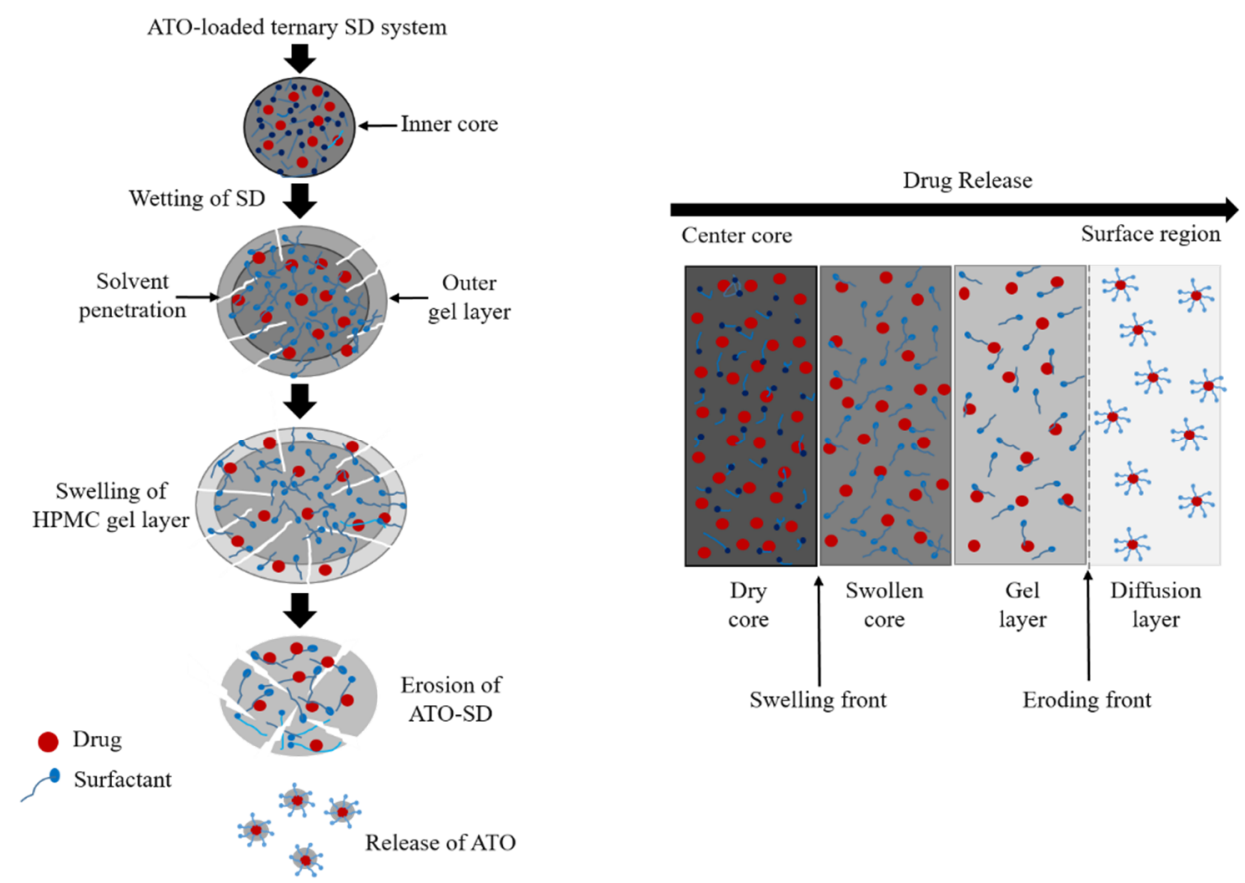

Figure 1. General schematic diagram showing drug release from a ternary solid dispersion with a hydroxypropyl methylcellulose (HPMC) carrier and a surfactant. Initial wetting hydrates the polymers and forms a gel layer around the SD. Penetration of water leads to expansion of the gel layer. Finally, as the water penetrates further into the core, the outer carrier layer becomes completely hydrated and dissolves, releasing the encapsulated drug.

\section{Materials and Methods}

\subsection{Materials}

ATO was kindly provided by Hanmi Pharm. Co. (Suwon, Korea). Hydroxypropyl methylcellulose (HPMC, 2910) and sodium lauryl sulfate (SLS) were purchased from Shin-Etsu Co. (Tokyo, Japan) and Duksan Chemical Co. (Ansan, Korea), respectively. All other chemicals and reagents were of HPLC-grade and were used without further purification.

\subsection{Screening of Excipients}

For the selection of a suitable solid matrix former, various hydrophilic polymers and surfactants were screened using solubility testing published guidelines with slight modifications [34]. An excess of drug was added to $1 \mathrm{~mL}$ of distilled water containing $1 \%(w / v)$ polymer or surfactant and vortexed for a few seconds. The microtubes were shaken at $25^{\circ} \mathrm{C}$ and $100 \mathrm{rpm}$ for five days in an isothermal water bath shaker. The resulting suspensions were centrifuged at 10,000 $\mathrm{g}$ for $10 \mathrm{~min}$ and the supernatant was filtered through a $0.45 \mu \mathrm{m}$ membrane filter. The filtrate was diluted with methanol as needed and then assayed using an Agilent 1260 Infinity LC system (Agilent Technologies; Santa Clara, CA, USA) equipped with Agilent ChemStation software (version B.04.02), an Agilent 1260 Quat pump, and an Capcell Pak C18 column (Shiseido, $250 \mathrm{~mm} \times 4.6 \mathrm{~mm}$ I.D., $5 \mu \mathrm{m}$ ). The mobile phase consisted of methanol, acetonitrile, and distilled water $(v / v / v: 40 / 40 / 20)$, at a flow rate of $1 \mathrm{~mL} / \mathrm{min}$. The mobile phase was monitored using an Agilent 1260 VWD detector (Agilent Technologies; Santa Clara, CA, 
USA) at a wavelength of $240 \mathrm{~nm}$, and the injection volume was $10 \mu \mathrm{L}$. All the experiments were carried out in triplicate $(n=3)$, and the solubility data are represented as mean \pm standard deviation.

\subsection{Preparation and Optimization of ATO-Loaded Solid Dispersions}

The polymer and surfactant demonstrating the highest aqueous drug solubility in screening tests were selected for investigating the optimal SD formulation. A Büchi B-290 nozzle-type mini spray dryer (Buchi Co.; Flawil, Switzerland) was employed for the preparation of ATO-loaded solid dispersions. Various ratios of HPMC and SLS (fixed at $0.1 \mathrm{~g}$ ) were dissolved in $300 \mathrm{~mL}$ of water. Then, $150 \mathrm{~mL}$ of ethanol containing $1 \mathrm{~g}$ of drug was added to the aqueous solution (Table 1). Each resulting solution was continuously stirred and transferred to a $0.7 \mathrm{~mm}$ pneumatic nozzle using a peristaltic pump and spray-dried, leading to production of ATO-loaded solid dispersions. The spray-drying conditions were inlet and outlet temperature of 90 and $69-71^{\circ} \mathrm{C}$, respectively; a feed rate of $10 \mathrm{~mL} / \mathrm{min}$; and $100 \%$ aspiration. A total of five SDs were prepared, and aqueous solubility testing of the drug in SDs was carried out to select the optimized formulation.

Table 1. Composition of various solid dispersions prepared using various polymer-to-surfactant ratios.

\begin{tabular}{cccc}
\hline Formulation & Drug $(\mathrm{g})$ & HPMC $(\mathrm{g})$ & SLS $(\mathrm{g})$ \\
\hline F1 & 1 & 0.5 & 0.1 \\
F2 & 1 & 1 & 0.1 \\
F3 & 1 & 2 & 0.1 \\
F4 & 1 & 4 & 0.1 \\
F5 & 1 & 8 & 0.1 \\
\hline
\end{tabular}

\subsection{Solubility of ATO in Solid Dispersions}

An excess amount of ATO-loaded SDs (equivalent to about approximately $100 \mathrm{mg}$ of free ATO) was added to distilled water, vortexed, and agitated at $25^{\circ} \mathrm{C}$ for five days. The resulting suspensions were centrifuged at 10,000 $\mathrm{g}$ for $10 \mathrm{~min}$ (Hanil; smart 15 plus), and the supernatant was filtered through a $0.45 \mu \mathrm{m}$ membrane filter and diluted with methanol. The concentration of ATO in the samples was assayed using HPLC as described above.

\subsection{In Vitro Dissolution Study}

The in vitro drug release of free drug, physical mixture, and optimized ATO-loaded SDs was carried out in a USP type II dissolution apparatus. The SD samples equivalent to $20 \mathrm{mg}$ of ATO were filled into hard gelatin capsules (size 0) and subjected to dissolution testing (ERWEKA; DT 620, Heusenstamm, Germany) with $900 \mathrm{~mL}$ of distilled water as the medium, maintained at a constant temperature $\left(37 \pm 0.5^{\circ} \mathrm{C}\right)$ with a paddle rotation speed of $100 \mathrm{rpm}$. In several publications involving ATO, water was used as a dissolution medium in the in vitro dissolution study [31,32], and therefore water was chosen as an in vitro dissolution medium in this work. At a predetermined time intervals $(5,10,15,30,45,60$, and $90 \mathrm{~min}), 2 \mathrm{~mL}$ of the medium was sampled and an identical amount of fresh dissolution medium was immediately replenished to compensate for the loss during sampling. Prior to analysis, the samples were filtered through a $0.45 \mu \mathrm{m}$ membrane filter. A $10 \mu \mathrm{L}$ aliquot of the sample was injected into the HPLC system and the concentration quantified using the method described above.

\subsection{Physicochemical Characterization}

\subsubsection{Loading Efficacy}

The SDs equivalent to $10 \mathrm{mg}$ ATO were completely dissolved in $100 \mathrm{~mL}$ methanol, filtered through $0.45 \mu \mathrm{m}$ membrane filter, and assayed for the content of drug by HPLC as mentioned above. The loading efficacy (\%) was calculated as follows; loading efficacy $(\%)=C_{\mathrm{a}} / C_{\mathrm{t}} \times 100$, where $C_{\mathrm{a}}$ and $C_{\mathrm{t}}$ were the actual and theoretical drug content in these SDs, respectively. 


\subsubsection{Scanning Electron Microscopy (SEM)}

The shape and surface morphology of the drug, carrier, surfactant, and SD formulation were examined using SEM (SU8220; Hitachi; Tokyo, Japan) operating at an accelerated voltage of $5.0 \mathrm{kV}$. Samples were affixed onto a brass specimen holder using double-sided adhesive tape, and the powders were made electrically conductive by coating with platinum $(6 \mathrm{~nm} / \mathrm{min})$ in vacuum $(0.8 \mathrm{~Pa})$ using an EmiTeck Sputter Coater K575 K (Quorum Technologies Ltd.; West Sussex, UK) for 4 min at 15 mA.

\subsubsection{Differential Scanning Calorimetry (DSC)}

The crystallinity of free ATO, carrier, surfactant, and SD were further investigated using a TA DSC Q20 instrument (TA Instruments; Newcastle, DE, USA). Approximately $5 \mathrm{mg}$ of sample was weighed, sealed, and placed in an aluminum pan. The samples were heated from $30^{\circ} \mathrm{C}$ to $220^{\circ} \mathrm{C}$ at a constant temperature change rate of $10^{\circ} \mathrm{C} / \mathrm{min}$ under nitrogen gas flow at $50 \mathrm{~mL} / \mathrm{min}$. A physical mixture (PM) was prepared by mixing the polymer, surfactant, and the drug at the same weight ratio as that of the optimized ATO-loaded SD (F2).

\subsubsection{X-ray Powder Diffraction}

X-ray powder diffraction (XRD) evaluation of the samples was done using a D/MAX-2500 XRD instrument (Rigaku, Japan) equipped with a copper anode operated using $\mathrm{Cu} \mathrm{K}_{\alpha}$ radiation $(1.54178 \AA$, $40 \mathrm{kV}$, and $40 \mathrm{~mA}$ ). The samples were scanned from $5^{\circ}$ to $35^{\circ}$ using step scan mode with a step size of $0.05^{\circ} / \mathrm{s}$ at room temperature and a $2 \theta$ diffraction angle to obtain the diffraction patterns.

\subsection{Pharmacokinetic Studies}

All animal care and procedures were performed in accordance with the guidelines and protocols approved by the Institutional Animal Care and Use Committee (IACUC) at Kyungpook National University (Permit Number: 2018-0129, Date of approval: 01 September 2018). The bioavailability of ATO in the SD was evaluated in 7-9-week-old, male Sprague Dawley rats, weighing 250-280 g (Samtako Bio Korea, Osan, Korea). The animals were randomly divided into two groups of six rats each. These rats were kept at controlled conditions of $25 \pm 2{ }^{\circ} \mathrm{C} / 55 \pm 5 \% \mathrm{RH}$ and fasted for $12 \mathrm{~h}$ prior to experiments with free access to water.

The rats were individually administered a $0.75 \mathrm{~mL}$ aqueous suspension of free ATO or SD (F2) equivalent to $30 \mathrm{mg} / \mathrm{kg}$ of ATO via oral gavage. Approximately $0.35 \mathrm{~mL}$ of blood was sampled via the jugular vein at predetermined time intervals and centrifuged at $8000 \mathrm{~g}$ for $10 \mathrm{~min}$ at $4{ }^{\circ} \mathrm{C}$ to separate the plasma. The plasma samples were stored at $-20^{\circ} \mathrm{C}$ until further studies. To $90 \mu \mathrm{L}$ of each plasma sample, $100 \mu \mathrm{L}$ of acetonitrile and $10 \mu \mathrm{L}$ of internal standard (ibuprofen in methanol, $100 \mu \mathrm{g} / \mathrm{mL}$ ) was added and vortexed for a few seconds. Then, the samples were centrifuged at 13,000 g for $10 \mathrm{~min}$ at $4{ }^{\circ} \mathrm{C}$ and the supernatants immediately transferred to vials for HPLC quantification. The optimized HPLC conditions [35] were used in this study. Briefly, a mobile phase composed of methanol, acetonitrile, and potassium dihydrogen phosphate (v/v/v: 20/50/30) adjusted to $\mathrm{pH} 3.5$ with orthophosphoric acid was used and the injection volume was $20 \mu \mathrm{L}$. The other HPLC conditions were the same as described in Section 2.2. All pharmacokinetic parameters, including area under the plasma concentration-time curve (AUC), maximum plasma concentration $\left(C_{\max }\right)$, time to reach the maximum plasma concentration $\left(T_{\max }\right)$, half-life $\left(t_{1 / 2}\right)$, and elimination rate constant $\left(K_{\mathrm{el}}\right)$ were estimated using WinNonlin ${ }^{\mathrm{TM}}$ (Pharsight Corp.; Mountain view, CA, USA). Values are reported as mean \pm S.D. and the data was statistically significant at $p<0.05$ between the two formulations checked by the Student's $t$ test. 


\section{Results and Discussion}

\subsection{Selection of Excipients}

Carrier selection plays a significant role in the performance of amorphous SDs, because the molecular dispersion of drug in the carrier must involve significant miscibility, with strong drug-carrier interactions (e.g., hydrogen-bonding) for stability against recrystallization [36,37]. To derive the maximal advantage from an ASD system, the carrier's miscibility with the drug should be investigated prior to formulation. The aqueous solubility of ATO in various carriers and surfactants is presented in Figure 2A. Among the carriers tested, HPMC showed the highest drug solubility at $385.82 \pm 37.78 \mu \mathrm{g} / \mathrm{mL}$, whereas PEG 6000 resulted in the lowest at $146.79 \pm 65.79 \mu \mathrm{g} / \mathrm{mL}$. Among the various surfactants tested, drug solubility in SLS was substantially high $(2587.92 \pm 210.01 \mu \mathrm{g} / \mathrm{mL})$ (Figure 2B). Therefore, HPMC and SLS were chosen as the hydrophilic carrier and surfactant for the formulation of ATO-loaded ASDs.

HPMC is nontoxic, chemically stable, and inert, and is therefore compatible with numerous drugs; further, it does not show pH-dependent solubility, and is a "generally regarded as safe" molecule, allowing its use in pharmacological applications [38,39]. The free energy of amorphous forms has excess enthalpy and entropy, which makes amorphous forms prone to recrystallization [40]. The addition of a surfactant was found to overcome the limitations associated with the formulation of a solid dispersion system such as, the poor miscibility of drug and polymer, recrystallization of drug in SD system, etc. [22]. Thus, the use of suitable matrix former is required to avoid the formation of stable drug crystals in a solid dispersion. Therefore, we used a low concentration of SLS ( $0.1 \mathrm{~g})$ as a surfactant along with the HPMC polymer in the formulated ternary ASDs system for enhancement of drug-polymer miscibility and stabilization of the amorphous state drug (prevent recrystallization of ATO) presented in the solid dispersions.

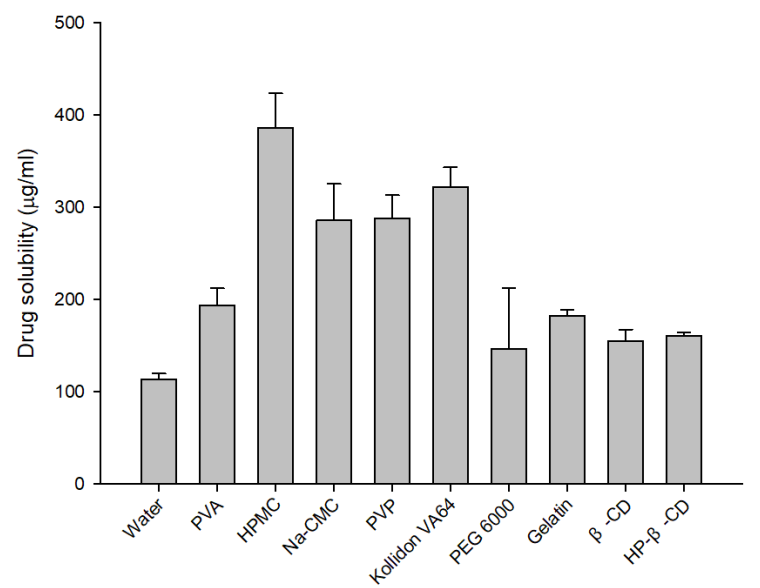

(A)

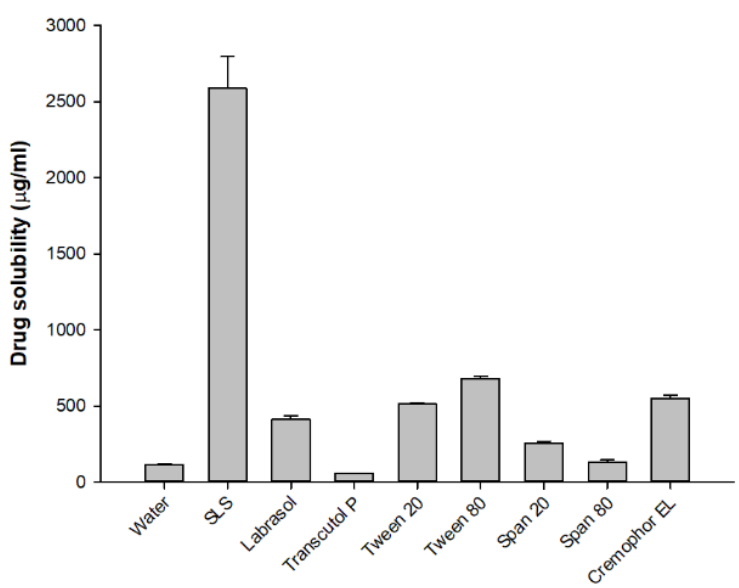

(B)

Figure 2. Solubility of atorvastatin calcium (ATO) in a different (A) polymer and (B) surfactant solutions $(1 \% w / v)(n=3)$.

\subsection{Optimization of ATO-Loaded SD}

The novel ATO-loaded ASD formulations were prepared using a lab-scale spray dryer with water and ethanol, a hydrophilic carrier (HPMC), and a surfactant (SLS). The prepared ATO-loaded ASDs were subjected to solubility studies to investigate the effect of the carriers on the drug solubility. The results in Figure 3A show that irrespective of the ratios of HPMC and SLS, a significant improvement (5.19-17.44-fold) in aqueous solubility of ATO was observed in all the formulations (F1-F5) compared to free drug alone. However, it was found that aqueous drug solubility did not improve with increasing HPMC concentration in the ASD formulations (F3-F5). This could be due to the highest level of supersaturation achieved with F2 formulation (Figure 3A). Once the maximum solubility is attained with 
F2, the further addition of polymer does not guarantee improved drug solubility which was seen with F3, F4, and F5 formulations. Similar results have been reported for atorvastatin [32], raloxifene [41], valsartan [42], etc. This suggested that choosing an optimal drug-carrier ratio is prerequisite to derive the maximal advantage from use of the ASD system. The highest drug solubilizing effect (i.e., supersaturation) was observed when the drug-carrier ratio was 1:1 (F2 formulation). Therefore, F2 was chosen as the optimized ATO-loaded ASD formulation for further studies.

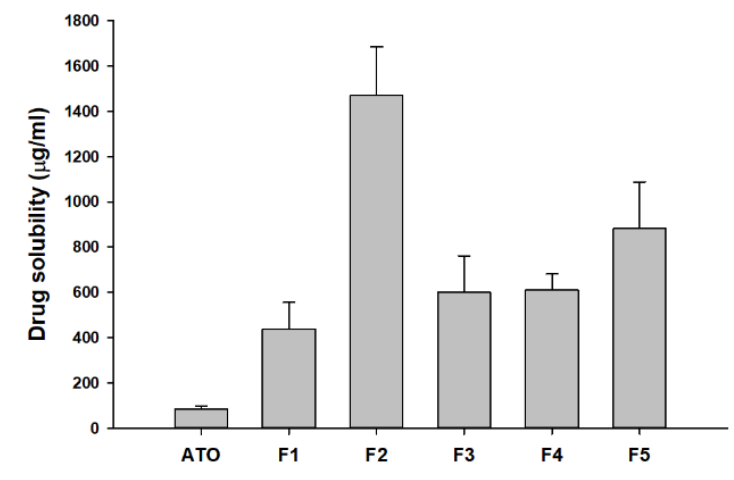

(A)

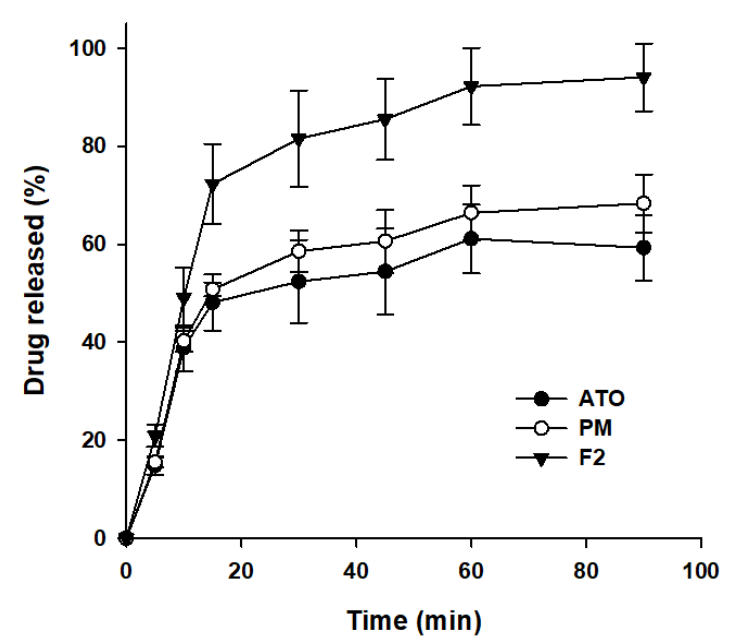

(B)

Figure 3. Solubility of ATO in various solid dispersions (SDs) (A) and dissolution profile of free ATO, PM, and F2 (B). The PM and F2 formulation was composed of ATO/HPMC/SLS at a weight ratio of 1:1:0.1. Each value represents the mean $\pm \operatorname{S.D}(n=3)$.

\subsection{Evaluation of ATO-Loaded SD}

The dissolution profile of free drug, physical mixture (PM), and the optimized ASD formulation (F2) was assessed using distilled water as dissolution medium. The dissolution profile of F2 showed an initial increase, with approximately $70 \%$ drug release within the first $15 \mathrm{~min}$, most probably due to an initial burst release of the hydrophobic drug from the hydrophilic matrix in ASD. One possible explanation is that the dissolution of F2 was accelerated due to the hydrophilic nature of HPMC, leading to rapid water transport into the SD matrix, which resulted in hydration, wetting, and drug release with much faster kinetics from the SD system. In addition, the presence of surfactant (SLS) in the ternary SD improved the drug-polymer miscibility and reduces the interfacial tension between the spray dried particles and the release media. Further, the increased in surface area available for dissolution due to the reduced particle size (observed in SEM image of F2 (Figure 4D)) of SDs would also have assisted in the initial burst drug release from the F2 formulation. Hence, the preparation of ATO-loaded SD essentially showed the initial burst release taking advantage of the increased surface area, amorphous state, and effective wettability of carriers (HPMC and SLS). Similar drug kinetic results have been reported for atorvastatin [30], atorvastatin calcium/ezetimibe [24], itraconazole [43], etc. By $60 \mathrm{~min}$, both the ATO and F2 had dissolved to their maximum state, showing plateaus thereafter. Moreover, F2 showed almost complete, i.e., 90\% dissolution within an hour, whereas free ATO showed approximately $60 \%$ dissolution within the same time frame (Figure 3B). In addition, PM showed improved drug dissolution than free drug yet lower than that of F2 formulation. The improved dissolution of ATO in physical mixture might be due to the presence of hydrophilic carriers (HPMC and SLS). Thus, the further increased in vitro dissolution of ATO-loaded SD (F2) compared to the bulk drug, as well as the PM, highlighted the significance of the solid dispersion system as a potential technique to enhance the biopharmaceutical characteristics of poorly water-soluble drugs. Overall, the SD formulation of ATO showed a significant increase in dissolution compared to the PM and free drug 
alone. This increase in dissolution can be explained by the enhanced supersaturation resulting from the molecular dispersion of the poorly water-soluble ATO in the hydrophilic carrier matrix.

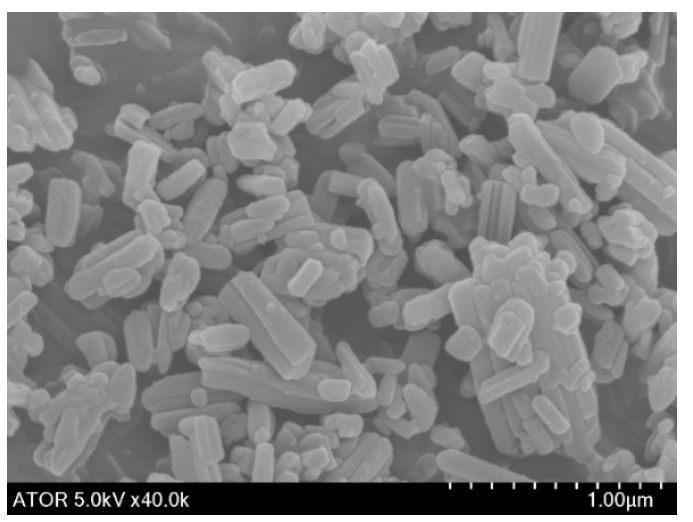

(A)

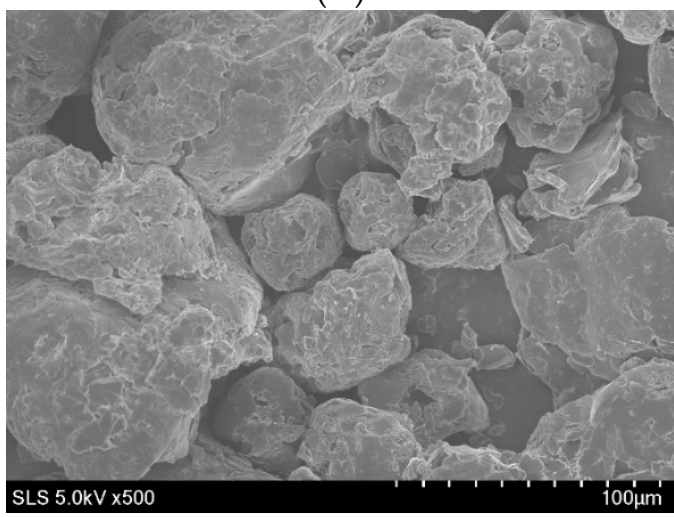

(C)

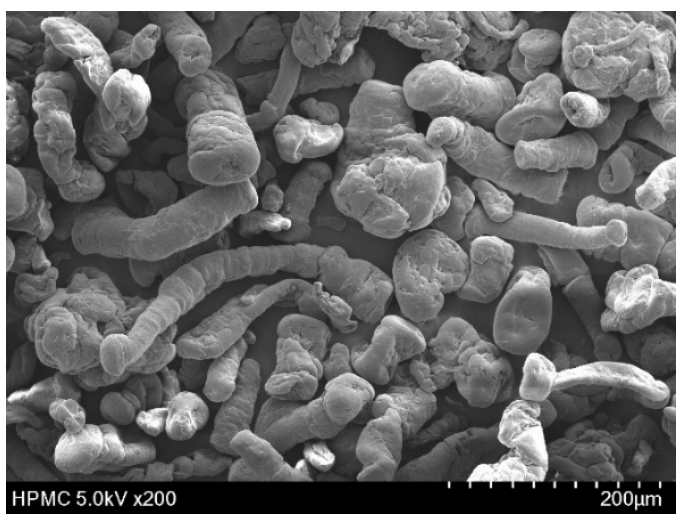

(B)

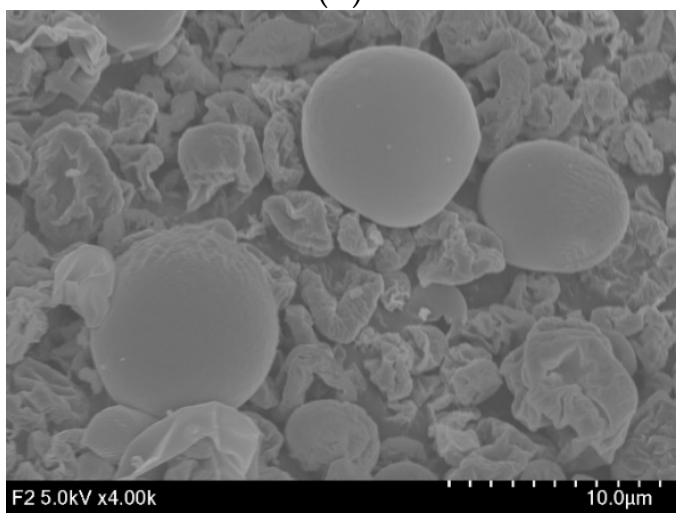

(D)

Figure 4. SEM images of (A) free ATO (40,000 X), (B) HPMC (200 X), (C) SLS (500 X), and (D) F2 $(4000 \mathrm{X})$.

\subsection{Physicochemical Characterization}

Physicochemical characterization is useful to examine the solid state properties of drug, carriers and spray-dried solid dispersions. The ATO-loaded SDs prepared in this study gave almost $100 \%$ loading efficiency. Scanning electron microscopy revealed the morphological characteristics of free ATO, HPMC, SLS, and F2 (Figure 4). The SEM image of free ATO showed slightly smooth-surfaced, long, cylindrical-shaped articles (Figure 4A), whereas that of F2 showed spherical shaped, smooth-surfaced solid dispersions (Figure 4D). In addition, the SEM images of HPMC and SLS (Figure 4B,C) featured larger rough and irregular-shaped particles. The presence of crystallinity could be anticipated by the appearance of drug particles on the outer surfaces of SDs [44]. As seen in the SEM image of Figure 4D, no observable drug particles were found on the outer surface of the F2, which could be due to the homogeneous dispersion of ATO within the carrier's matrix. Interestingly, we could also notice several small-sized SDs with small dents on the outer surface, which could have formed due to the rapid solvent evaporation during the spray-drying process. Based on these observations, it appears that the bulk drug was dispersed into the amorphous carriers in ATO-loaded SDs. Further, DSC and PXRD were employed to confirm the amorphous state of the ATO-loaded SD system.

DSC was used to investigate the thermal behavior of free ATO, HPMC, SLS, and F2 (Figure 5A). Thermal analysis is a commonly used and useful tool, providing information regarding melting, phase transition, recrystallization, chemical degradation, and change in the specific heat capacity, and is useful for understanding the physiochemical characteristics of the dispersed drug inside the carriers [45]. Free ATO showed an endothermic peak at approximately $175^{\circ} \mathrm{C}$, corresponding to its melting point and indicating its crystalline nature [46]. Moreover, HPMC showed a broad endotherm at approximately 
60-130 ${ }^{\circ} \mathrm{C}$ corresponding to its glass transition temperature $\left(T_{\mathrm{g}}\right)$ [47], and SLS showed a sharp distinct endothermic peak at approximately $190^{\circ} \mathrm{C}$ (melting) with a small broad peak at approximately $90{ }^{\circ} \mathrm{C}$ (loss of water) $[48,49]$. Further, PM showed an endotherm corresponding to the peaks of ATO, HPMC, and SLS, but with reduced intensity, indicating the presence of crystallinity and the absence of any strong interaction between drug and carriers. On the contrary, F2 did not produce any characteristic peaks in the DSC thermogram related to the bulk drug, which indicates the transformation of crystalline state ATO into its amorphous form in SD. Interestingly, a single broad endotherm peak with reduced intensity was observed for $\mathrm{F} 2$ at around $50-100{ }^{\circ} \mathrm{C}$. This peak corresponds to the glass transition temperature $\left(T_{\mathrm{g}}\right)$ observed due to the presence of HPMC in amorphous SD (Figure 5). However, the $T_{\mathrm{g}}$ value was broadened and shifted to a lower temperature range, presumably as a consequence of moisture loss from the F2 formulation. Similar peaks have been reported previously in studies using HPMC [47]. Generally, the presence of moisture lowers the temperature at which the transition occurs and also broadens the $T_{\mathrm{g}}$ range over which is it seen. These DSC results suggest a strong interaction between the drug and carriers due to molecular dispersion of drug into carrier matrix, which may have resulted in the amorphous nature of the ATO-loaded solid dispersion, and therefore in the absence of the drug characteristic melting peak.

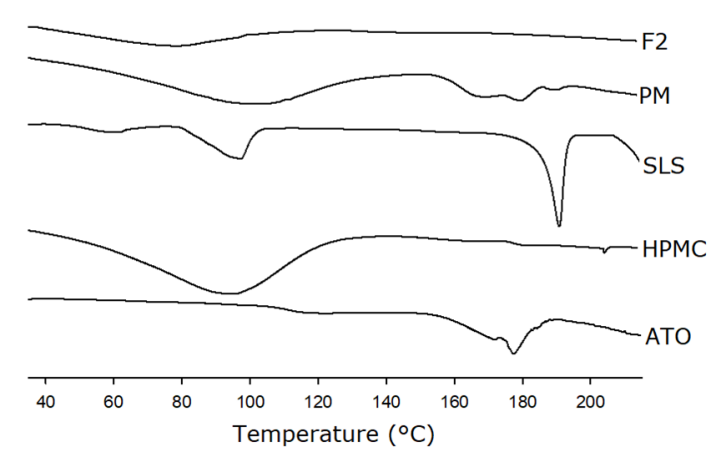

(A)

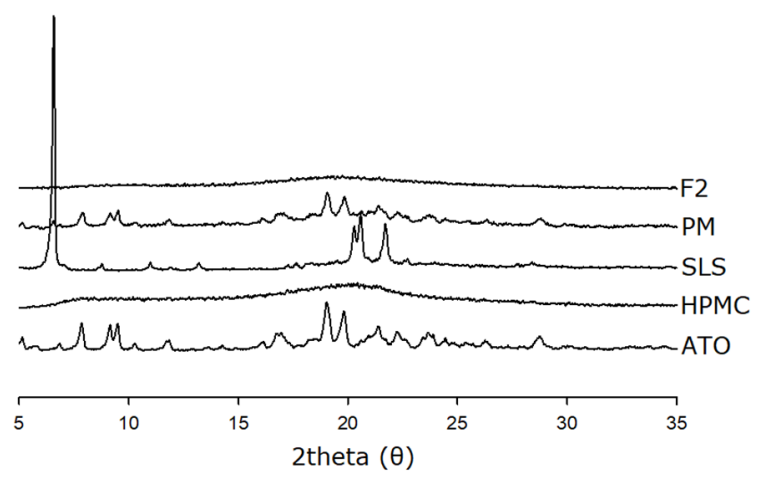

(B)

Figure 5. DSC (A) and XRD (B) curves of samples. PM represents a physical mixture of ATO, HPMC, and SLS at a 1:1:0.1 (w/w/w) ratio.

Additionally, PXRD was conducted to further elucidate the amorphous nature of the SD. Figure 5B illustrates the PXRD patterns of free ATO, HPMC, SLS, PM, and F2. The diffraction pattern of free drug showed numerous sharp peaks, mostly at approximately $7^{\circ}-30^{\circ}$ of $2 \theta$, suggesting highly crystalline characteristics of ATO [31]. HPMC and SLS showed numerous intrinsic peaks, and, in particular, SLS showed high-intensity peaks at approximately $5^{\circ}$ and above $20^{\circ}$, due to its crystalline nature. Additionally, X-ray diffraction pattern of PM showed most of the major characteristic peaks as in those of the pure drug, HPMC, and SLS, suggesting its crystalline nature. In contrast, no characteristic diffraction peaks corresponding to free drug or carriers were present in F2. Thus, absence of any diffraction peaks in F2 is further evidence of the amorphous nature of ATO in the ATO-loaded SD, consistent with the DSC and SEM results.

Stable crystal lattice of drug molecules shows problems in solubilization due to its higher lattice energy. The reason for improved solubility and in vitro dissolution rate could be associated with the amorphous nature of ASDs (F2). In the amorphous form, molecules have the highest free energy and entropy resulting in superior molecular mobility; therefore, it requires less external energy for dissolution compared to its crystalline counterpart [16]. Therefore, changing the solid-state properties of drugs from crystalline to amorphous forms renders distinct advantage with regards to solubility. 


\subsection{Pharmacokinetic Studies}

The bioavailability of ATO in ternary ASD was assessed in rats. Figure 6 shows the mean plasma concentration versus time profiles of ATO after oral administration of free ATO or ATO-loaded SD (F2) at a dose of $30 \mathrm{mg} / \mathrm{kg}$ ATO. (cf. Figure S1 Supporting Information). The pharmacokinetic parameters are listed in Table 2. As shown in Figure 6, the total plasma concentrations of ATO were significantly higher in rats administered the F2 formulation than in those administered free drug powder alone, at all time points. The increase in bioavailability of a hydrophobic drug tends to be directly proportional to increase in overall bioavailability. The maximal ATO plasma concentration $\left(C_{\max }\right)$ due to ATO-loaded SD (F2) $(786.23 \pm 84.72 \mathrm{ng} / \mathrm{mL})$ was remarkably higher than that due to free ATO $(328.83 \pm 46.56 \mathrm{ng} / \mathrm{mL})$ (Table 2). In addition, the AUC of free drug and the F2 formulation was $1557.34 \pm 221.46 \mathrm{~h} \cdot \mathrm{ng} / \mathrm{mL}$ and $2621.60 \pm 318.99 \mathrm{~h} \cdot \mathrm{ng} / \mathrm{mL}$, respectively. Both the $C_{\max }$ and AUC of ATO-SD were approximately 2.4and 1.68-fold higher compared to those of free ATO. However, there was no significant differences in the $T_{\max }, t_{1 / 2}$, or $K_{\mathrm{el}}$ values of free drug and F2. These data show that use of a ternary ATO-loaded ASD results in substantial improvement of ATO absorption, possibly due to increased ATO solubility and dissolution rate in the gastrointestinal tract, and the amorphous nature of ATO in ASD. In clinical practice, in order to attain the same level of pharmacotherapeutic actions with ATO, the therapeutic dose of ATO in ATO-loaded SD could be now reduced by 1.68-fold. Hence, this could offer several benefits, including reduction in dose intake by patients (generally higher amount of API) and excipients associated with conventional oral formulations, fewer side effects than conventional dosages, and improved cost-effectiveness and overall patience compliance.

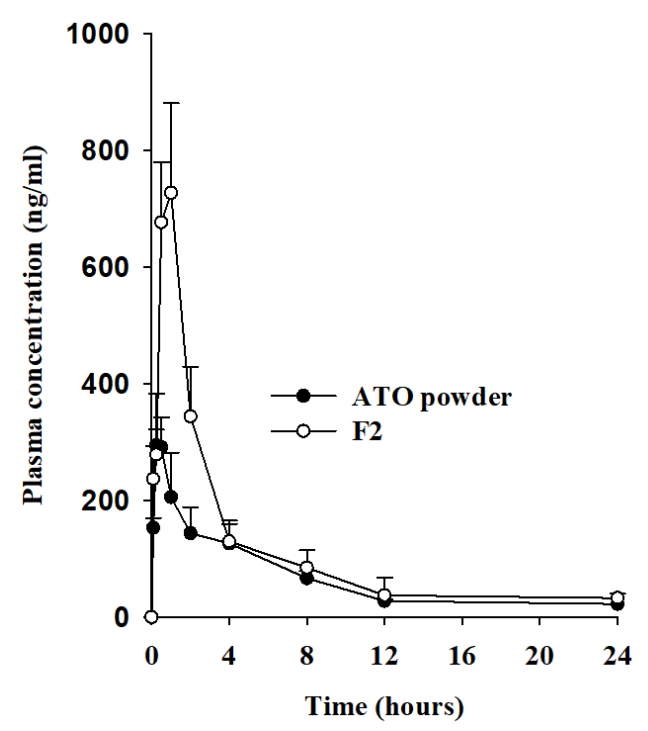

Figure 6. Plasma concentration-time profiles of ATO after oral administration of free drug or solid dispersions in rats. Each value represents the mean \pm S.D. $(n=6) .{ }^{*} p<0.05$ compared to free ATO.

Table 2. Pharmacokinetic parameters.

\begin{tabular}{ccc}
\hline Formulations & ATO & F2 \\
\hline AUC $(\mathrm{h} \cdot \mathrm{ng} / \mathrm{mL})$ & $1557.34 \pm 221.46$ & $2,621.60 \pm 318.99 *$ \\
$C_{\max }(\mathrm{ng} / \mathrm{mL})$ & $328.83 \pm 46.56$ & $786.23 \pm 84.72^{*}$ \\
$T_{\max }(\mathrm{h})$ & $0.40 \pm 0.14$ & $0.70 \pm 0.27$ \\
$t_{1 / 2}(\mathrm{~h})$ & $4.15 \pm 0.54$ & $4.64 \pm 1.45$ \\
$K_{\mathrm{el}}\left(\mathrm{h}^{-1}\right)$ & $0.17 \pm 0.02$ & $0.17 \pm 0.09$ \\
\hline
\end{tabular}

\footnotetext{
${ }^{*} p<0.05$ compared with free ATO. Each value represents the mean \pm S.D. $(n=6)$
} 


\section{Conclusions}

In the present study, hydrophilic polymer was successfully used to encapsulate the hydrophobic drug ATO into the solid dispersions via a spray-drying technique. We found increased solubility and improved dissolution profile of ATO in the prepared SD, due to efficient molecular dispersion of ATO in the hydrophilic carrier matrix and the conversion of the drug from a crystalline state to its higher energy amorphous form in the SD. The presence of only a single $T_{\mathrm{g}}$ observed over the entire DSC scan of F2 indicates good drug carrier miscibility in the prepared ASD. The pharmacokinetic parameters of the ATO-loaded SD were significantly higher than those of the free drug because of increased bioavailability, largely due to enhanced drug concentrations in the gastrointestinal (GI) tract. Hence, this could offer numerous benefits in a clinical setting, including the reduction in dose intake by patients (generally, a higher amount of API and excipients is associated with conventional oral formulations), resulting in fewer side effects and greater cost-effectiveness, and leading to improved patience compliance. Overall, our in vitro data, the results of the physical characterization of the SDs, along with supporting in vivo data show significant potential in the use of HPMC as a hydrophilic matrix-former for amorphous solid dispersion systems to enhance biopharmaceutical performance of poorly water-soluble drugs.

Supplementary Materials: The following are available online at http://www.mdpi.com/1999-4923/11/9/461/s1, Figure S1: Plasma concentration (log scale)-time profiles of ATO after oral administration of free drug or solid dispersions in rats.

Author Contributions: Conceptualization, J.K., E.S.S., and D.W.K.; methodology, J.K. and D.W.K.; investigation, J.K., E.S.S., J.B., J.L., and D.W.K.; writing-original draft preparation, J.K., B.R.G., and D.W.K.; writing-review and editing, B.R.G. and D.W.K.; supervision, project administration, and funding acquisition, D.W.K.

Funding: This research was supported by the National Research Foundation (NRF) of South Korea grants funded by the South Korean government (MEST) (No. 2018R1D1A1B07050598).

Conflicts of Interest: The authors declare no conflicts of interest.

\section{References}

1. Hauss, D.J. Oral lipid-based formulations. Adv. Drug Deliv. Rev. 2007, 59, 667-676. [CrossRef] [PubMed]

2. Lindenberg, M.; Kopp, S.; Dressman, J.B. Classification of orally administered drugs on the World Health Organization Model list of Essential Medicines according to the biopharmaceutics classification system. Eur. J. Pharm. Biopharm. 2004, 58, 265-278. [CrossRef]

3. Serajuddin, A.T.M. Salt formation to improve drug solubility. Adv. Drug Deliv. Rev. 2007, 59, 603-616. [CrossRef]

4. Stella, V.J.; Nti-Addae, K.W. Prodrug strategies to overcome poor water solubility. Adv. Drug Deliv. Rev. 2007, 59, 677-694. [CrossRef]

5. Brittain, H.G. Effects of mechanical processing on phase composition. J. Pharm. Sci. 2002, 91, 1573-1580. [CrossRef]

6. Loftsson, T. Drug solubilization by complexation. Int. J. Pharm. 2017, 531, 276-280. [CrossRef] [PubMed]

7. Vadlamudi, H.C.; Yalavarthi, P.R.; Nagaswaram, T.; Rasheed, A.; Peesa, J.P. In-vitro and pharmacodynamic characterization of solidified self microemulsified system of quetiapine fumarate. J. Pharm. Investig. 2019, 49, 161-172. [CrossRef]

8. Liu, X.; Feng, X.; Williams, R.O.; Zhang, F. Characterization of amorphous solid dispersions. J. Pharm. Investig. 2018, 48, 19-41. [CrossRef]

9. McFall, H.; Sarabu, S.; Shankar, V.; Bandari, S.; Murthy, S.N.; Kolter, K.; Lanley, N.; Kim, D.W.; Repka, M.A. Formulation of aripiprazole-loaded $\mathrm{pH}$-modulated solid dispersions via hot-melt extrusion technology: In vitro and in vivo studies. Int. J. Pharm. 2019, 554, 302-311. [CrossRef]

10. Park, J.H.; Cho, J.H.; Kim, D.S.; Kim, J.S.; Din, F.U.; Kim, J.O.; Yong, C.S.; Youn, Y.S.; Oh, K.T.; Kim, D.W.; et al. Revaprazan-loaded surface-modified solid dispersion: Physicochemical characterization and in vivo evaluation. Pharm. Dev. Technol. 2019, 4, 788-793. [CrossRef] 
11. Sekiguchi, K.; Obi, N. Studies on Absorption of Eutectic Mixture. I. A comparison of the behavior of eutectic mixture of sulfathiazole and that of ordinary sulfathiazole in man. Chem. Pharm. Bull. 1961, 9, 866-872. [CrossRef]

12. Sekiguchi, K.; Obi, N.; Ueda, Y. Studies on absorption of eutectic mixture. II. Absorption of fused conglomerates of chloramphenicol and urea in rabbits. Chem. Pharm. Bull. 1964, 12, 134-144. [CrossRef] [PubMed]

13. Tanaka, R.; Hattori, Y.; Horie, Y.; Kamada, H.; Nagato, T.; Otsuka, M. Characterization of Amorphous Solid Dispersion of Pharmaceutical Compound with $\mathrm{pH}$-Dependent Solubility Prepared by Continuous-Spray Granulator. Pharmaceutics 2019, 11, 159. [CrossRef]

14. Bikiaris, D.N. Solid dispersions, part I: Recent evolutions and future opportunities in manufacturing methods for dissolution rate enhancement of poorly water-soluble drugs. Expert Opin. Drug Deliv. 2011, 8, 1501-1519. [CrossRef] [PubMed]

15. Willart, J.F.; Descamps, M. Solid state amorphization of pharmaceuticals. Mol. Pharm. 2008, 5, 905-920. [CrossRef] [PubMed]

16. Patel, B.B.; Patel, J.K.; Chakraborty, S.; Shukla, D. Revealing facts behind spray dried solid dispersion technology used for solubility enhancement. Saudi Pharm. J. 2015, 23, 352-365. [CrossRef] [PubMed]

17. Ganesan, P.; Soundararajan, R.; Shanmugam, U.; Ramu, V. Development, characterization and solubility enhancement of comparative dissolution study of second generation of solid dispersions and microspheres for poorly water soluble drug. Asian J. Pharm. Sci. 2015, 10, 433-441. [CrossRef]

18. Jain, A.K.; Söderlind, E.; Viridén, A.; Schug, B.; Abrahamsson, B.; Knopke, C.; Tajarobi, F.; Blume, H.; Anschütz, M.; Welinder, A.; et al. The influence of hydroxypropyl methylcellulose (HPMC) molecular weight, concentration and effect of food on in vivo erosion behavior of HPMC matrix tablets. J. Control. Release 2014, 187, 50-58. [CrossRef] [PubMed]

19. Ghimire, M.; Hodges, L.A.; Band, J.; O’Mahony, B.; McInnes, F.J.; Mullen, A.B.; Stevens, H.N.E. In-vitro and in-vivo erosion profiles of hydroxypropylmethylcellulose (HPMC) matrix tablets. J. Control. Release 2010, 147, 70-75. [CrossRef]

20. Dahlberg, C.; Fureby, A.; Schuleit, M.; Dvinskikh, S.V.; Furó, I. Polymer mobilization and drug release during tablet swelling. A 1H NMR and NMR microimaging study. J. Control. Release 2007, 122, 199-205. [CrossRef]

21. Paudel, A.; Worku, Z.A.; Meeus, J.; Guns, S.; Van den Mooter, G. Manufacturing of solid dispersions of poorly water soluble drugs by spray drying: Formulation and process considerations. Int. J. Pharm. 2013, 453, 253-284. [CrossRef] [PubMed]

22. Chaudhari, S.P.; Dugar, R.P. Application of surfactants in solid dispersion technology for improving solubility of poorly water soluble drugs. J. Drug Deliv. Sci. Technol. 2017, 41, 68-77. [CrossRef]

23. Feng, D.; Peng, T.; Huang, Z.; Singh, V.; Shi, Y.; Wen, T.; Lu, M.; Quan, G.; Pan, X.; Wu, C. Polymer-surfactant system based amorphous solid dispersion: Precipitation inhibition and bioavailability enhancement of itraconazole. Pharmaceutics 2018, 10, 53. [CrossRef] [PubMed]

24. Jahangiri, A.; Barzegar-Jalali, M.; Garjani, A.; Javadzadeh, Y.; Hamishehkar, H.; Asadpour-Zeynali, K.; Adibkia, K. Evaluation of physicochemical properties and in vivo efficiency of atorvastatin calcium/ezetimibe solid dispersions. Eur. J. Pharm. Sci. 2016, 82, 21-30. [CrossRef] [PubMed]

25. Desager, J.P.; Horsmans, Y. Clinical pharmacokinetics of 3-hydroxy-3-methylglutaryl-coenzyme A reductase inhibitors. Clin. Pharmacokinet 1996, 31, 348-371. [CrossRef] [PubMed]

26. Malhotra, H.S.; Goa, K.L. Atorvastatin: An Updated Review of Its Pharmacological Properties and Use in Dyslipidaemia. Drugs 2001, 61, 1835-1881. [CrossRef]

27. Lea, A.P.; McTavish, D. Atorvastatin: A Review of Its Pharmacology and Therapeutic Potential in the Management of Hyperlipidemias. Drugs 1997, 53, 828-847. [CrossRef]

28. Cilla, D.D.J.; Whitfield, L.R.; Gibson, D.M.; Sedman, A.J.; Posvar, E.L. Multiple-dose pharmacokinetics, pharmacodynamics, and safety of atorvastatin, an inhibitor of HMG-CoA reductase, in healthy subjects. Clin. Pharmacol. Ther. 1996, 60, 687-695. [CrossRef]

29. Corsini, A.; Bellosta, S.; Baetta, R.; Fumagalli, R.; Paoletti, R.; Bernini, F. New insights into the pharmacodynamic and pharmacokinetic properties of statins. Pharmacol. Ther. 1999, 84, 413-428. [CrossRef]

30. Dong, W.; Su, X.; Xu, M.; Hu, M.; Sun, Y.; Zhang, P. Preparation, characterization, and in vitro/vivo evaluation of polymer-assisting formulation of atorvastatin calcium based on solid dispersion technique. Asian J. Pharm. Sci. 2018, 13, 546-554. [CrossRef] 
31. Ha, E.S.; Baek, I.H.; Cho, W.; Hwang, S.J.; Kim, M.S. Preparation and Evaluation of Solid Dispersion of Atorvastatin Calcium with Soluplus ${ }^{\circledR}$ by Spray Drying Technique. Chem. Pharm. Bull. 2014, 62, 545-551. [CrossRef] [PubMed]

32. Sarker, M.S.; Barman, R.K.; Ali, M.A.; Noguchi, S.; Iwao, Y.; Itai, S.; Wahed, M.I.I. Formulation Development and In-Vivo Evaluation of Atorvastatin Calcium Solid Dispersion in Streptozotocin Induced Diabetic Mice. Pharmacol. Pharm. 2018, 9, 395. [CrossRef]

33. Arunkumar, N.; Deecaraman, M.; Rani, C.; Mohanraj, K.P.; Venkateskumar, K. Formulation development and in vitro evaluation of nanosuspensions loaded with Atorvastatin calcium. Asian J. Pharm. 2014, 4. [CrossRef]

34. Connors, K.A.; Higuchi, T. Phase Solubility Techniques. Adv. Anal. Chem. Instrum. 1965, 4, 117-212.

35. Shah, D.A.; Bhatt, K.K.; Shankar, M.B.; Mehta, R.S.; Gandhi, T.R.; Baldania, S.L. RP-HPLC determination of atorvastatin calcium and amlodipine besylate combination in tablets. Indian J. Pharm. Sci. 2006, 68, 796-799. [CrossRef]

36. Medarević, D.; Djuriš, J.; Barmpalexis, P.; Kachrimanis, K.; Ibrić, S. Analytical and computational methods for the estimation of drug-polymer solubility and miscibility in solid dispersions development. Pharmaceutics 2019, 11, 372. [CrossRef] [PubMed]

37. Pereira, J.M.; Mejia-Ariza, R.; Ilevbare, G.A.; McGettigan, H.E.; Sriranganathan, N.; Taylor, L.S.; Davis, R.M.; Edgar, K.J. Interplay of degradation, dissolution and stabilization of clarithromycin and its amorphous solid dispersions. Mol. Pharm. 2013, 10, 4640-4653. [CrossRef]

38. Burdock, G.A. Safety assessment of hydroxypropyl methylcellulose as a food ingredient. Food Chem. Toxicol. 2007, 45, 2341-2351. [CrossRef]

39. Jaipal, A.; Pandey, M.M.; Charde, S.Y.; Raut, P.P.; Prasanth, K.V.; Prasad, R.G. Effect of HPMC and mannitol on drug release and bioadhesion behavior of buccal discs of buspirone hydrochloride: In-vitro and in-vivo pharmacokinetic studies. Saudi Pharm. J. 2015, 23, 315-326. [CrossRef]

40. Graeser, K.A.; Patterson, J.E.; Zeitler, J.A.; Rades, T. The role of configurational entropy in amorphous systems. Pharmaceutics 2010, 2, 224-244. [CrossRef]

41. Tran, T.H.; Poudel, B.K.; Marasini, N.; Woo, J.S.; Choi, H.G.; Yong, C.S.; Kim, J.O. Development of raloxifene-solid dispersion with improved oral bioavailability via spray-drying technique. Arch. Pharmacal Res. 2013, 36, 86-93. [CrossRef]

42. Yan, Y.D.; Sung, J.H.; Kim, K.K.; Kim, D.W.; Kim, J.O.; Lee, B.J.; Yong, C.S.; Choi, H.G. Novel valsartan-loaded solid dispersion with enhanced bioavailability and no crystalline changes. Int. J. Pharm. 2012, 422, 202-210. [CrossRef]

43. Kapsi, S.G.; Ayres, J.W. Processing factors in development of solid solution formulation of itraconazole for enhancement of drug dissolution and bioavailability. Int. J. Pharm. 2001, 229, 193-203. [CrossRef]

44. Goyanes, A.; Fina, F.; Martorana, A.; Sedough, D.; Gaisford, S.; Basit, A.W. Development of modified release 3D printed tablets (printlets) with pharmaceutical excipients using additive manufacturing. Int. J. Pharm. 2017, 527, 21-30. [CrossRef]

45. Le, T.T.; Elyafi, E.; Khaliq, A.; Mohammed, A.R.; Al-Khattawi, A. Delivery of Poorly Soluble Drugs via Mesoporous Silica: Impact of Drug Overloading on Release and Thermal Profiles. Pharmaceutics 2019, 11, 269. [CrossRef]

46. Panghal, D.; Nagpal, M.; Thakur, G.S.; Arora, S. Dissolution improvement of atorvastatin calcium using modified locust bean gum by the solid dispersion technique. Sci. Pharm. 2013, 82, 177-192. [CrossRef]

47. Ford, J.L. Thermal analysis of hydroxypropylmethylcellulose and methylcellulose: Powders, gels and matrix tablets. Int. J. Pharm. 1999, 179, 209-228. [CrossRef]

48. Craye, G.; Löbmann, K.; Grohganz, H.; Rades, T.; Laitinen, R. Characterization of amorphous and co-amorphous simvastatin formulations prepared by spray drying. Molecules 2015, 20, 21532-21548. [CrossRef]

49. Freire, F.D.; Aragão, C.F.S.; Moura, T.; Raffin, F.N. Compatibility study between chlorpropamide and excipients in their physical mixtures. J. Therm. Anal. Calorim. 2009, 97, 355. [CrossRef]

(C) 2019 by the authors. Licensee MDPI, Basel, Switzerland. This article is an open access article distributed under the terms and conditions of the Creative Commons Attribution (CC BY) license (http://creativecommons.org/licenses/by/4.0/). 